\title{
Do Older People Benefit from Having a Confidant? An Oklahoma Physicians Resource/Research Network (OKPRN) Study
}

\author{
Frank H. Lawler, MD, MSPH, James W. Mold, MD, MPH, and \\ Laine H. McCarthy, MLIS
}

Objective: The objective was to determine whether having a confidant was associated with improved health-related quality of life (HRQOL) or survival in older, community-dwelling individuals.

Methods: This prospective cohort study included 23 family physician members of the Oklahoma Physicians Research/Resource Network in 9 practices and 852 community-dwelling adults 65 or older participating in the Oklahoma Longitudinal Assessment of Health Outcomes of Mature Adults Studies. Longitudinal models analyzed changes in self-administered Quality of Well-Being (QWB-SA) scores over an average (S.D.) of 2.51 (1.28) years. Cox proportional hazards models assessed variables possibly associated with mortality over an average survival time (+/-S.D.) of 9.22 (3.24) years. We controlled for chronic illnesses, baseline age, gender, marital status, income, race, BMI, education and specified Medical Outcomes Study Short Form-36 (SF-36) domain scores.

Results: Initially, 740 participants $(87 \%)$ had a confidant. Being married was strongly associated with having a confidant $(91.9 \%$ vs. $77.8 \%, \mathbf{p}<0.0001)$. A confidant was associated with better SF-36 domain scores $(\mathrm{p}<0.0001)$, less morbidity, higher baseline QWB-SA scores and favorable changes in QWB-SA ( $<<0.0001)$. Unadjusted risk of death $(37.8 \%$ vs $46.4 \%, p=0.08)$ was not lower. Kaplan-Meier confidant status survival curves were not statistically different $(p=0.16)$.

Conclusions: Older people with a confidant demonstrated enhanced HRQOL maintenance over the short term, but not greater survival. (J Am Board Fam Med 2013;26:9-15.)

Keywords: Aged, Elderly, Interpersonal Relations, Practice-based Research, Primary Health Care

A confidant has been defined as "someone with whom the patient has regular contact (at least once a month) and with whom he or she can share sensitive personal information and gain support." This may be a spouse, a child, a friend, or a sibling-anyone who is trusted with one's deepest thoughts and feelings.

This article was externally peer reviewed.

Submitted 29 February 2012; revised 29 August 2012; accepted 10 September 2012.

From the Department of Family Medicine, University of Oklahoma, Oklahoma City.

Funding: Support for this study was provided by the Presbyterian Health Foundation and the University of Oklahoma Department of Family and Preventive Medicine.

Conflict of interest: none declared.

Corresponding author: Frank Lawler, MD, MSPH, Department of Family Medicine, University of Oklahoma, 900 NE 10th St, Oklahoma City, OK 73104 (E-mail: flawlermd@sib.ok.gov).
Most studies of the impact of a confidant on health outcomes have focused on population subgroups with specific disease states or have not controlled for important confounders such as severity of illness or functional status. For example, a prospective cohort of 674 elderly, communitydwelling participants found that those with no social contact with friends or family during the 2 weeks before a hip fracture had a 5 times greater risk of death during the following 2-year period than those who had daily social contact. ${ }^{2}$ A prospective study of 589 individuals monitored for 12 months after myocardial infarction showed that lack of a confidantnot previous depression-was associated with an adverse outcome after myocardial infarction. ${ }^{1}$ Loneliness and living alone may be concomitant with the lack of a confidant and have been shown to be predictors of functional decline and death among older people., ${ }^{3,4}$ The Reduction of Atherothrombosis for Continued 
Health (REACH) study found that living alone was associated with higher 4-year mortality $(14.1 \%$ vs $11.1 \% ; P<.01)$ and cardiovascular death $(8.6 \%$ vs $6.8 \% ; P<.01) .5$ A Spanish cohort of 1174 elderly residents with a confidant had a $25 \%$ (95\% CI, 5-40) reduction in mortality. Those results were similar for both men and women. ${ }^{6}$

The primary purpose of this analysis of data was to determine whether having a confidant was associated with better subsequent health-related quality of life, improved survival, or both in a cohort of older people with and without physical disabilities.

\section{Methods}

The data used in this study were obtained from the data set of the Oklahoma Longitudinal Assessment of the Health Outcomes of Mature Adults (OKLAHOMA) studies. Previous publications have described in greater detail the methodology used to obtain this data. ${ }^{7,8}$ This study was reviewed and approved by the institutional review board of the University of Oklahoma Health Sciences Center, and all participants consented to take part, first by phone and then by signing a written consent form.

Between January 1, 1999, and December 31, 2000, 23 family physician members of the Oklahoma Physicians Resource/Research Network in 9 practices statewide created, from their billing records, lists of patients $\geq 65$ years of age seen by them within the past 18 months. Patients were excluded if they had switched physicians, died, were in nursing homes, or the patient's primary care physician felt the patient was too confused to sign consent. Eligible patients received a letter from their physician inviting them to participate. Two weeks later, the project coordinator contacted these patients via telephone. Those who agreed to participate were asked to complete a questionnaire that was mailed to them before their enrollment visit.

The questionnaire included questions about demographic information, health habits, symptoms, medical conditions, activities of daily living skills (ADLs; 14-point scale), instrumental ADLs (IADLs; 14-point scale), the Medical Outcomes Study 36-item short form (SF-36), ${ }^{9}$ self-rated health (5-point Likert and 100-point rating scales), and the self-administered Quality of Well-Being (QWB-SA) instruments. Participants also were asked a single yes/no item, similar to questions used in other studies, ${ }^{10,11}$ investigating the availability of a confidant: "Do you have someone to whom you can tell your deepest thoughts and feelings?"

Two research nurses enrolled participants at their family physicians' offices at times scheduled specifically for this purpose. The nurses reviewed the study protocol, obtained informed consent, and checked the questionnaire for completeness. Each year on the anniversary of their initial enrollment, participants were invited to re-enroll. Those who agreed again provided consent to participate and completed a follow-up questionnaire. The number of participants who enrolled in year 1 was $852 ; 597$, 401 , and 319 patients re-enrolled in years 2, 3, and 4 , respectively. Confidant status was assessed each year using the same question.

Participant deaths were determined at the end of the 5-year study using information provided by their designated contacts, their primary care physicians, and the Social Security Death Index (SSDI; http://ssdi.rootsweb.ancestry.com). Subsequent deaths have been tracked using the SSDI.

To reduce the expected impact of severity of illness on potential associations between confidant status and outcomes, 2 subgroups were created based on functional status. Because of a large skew to the left, we dichotomized functional status into those with total ADLs plus IADLs scores of $\geq 27$ and all others. The choice of a cutoff at 27 rather than 28 allowed those with urinary incontinence to be included in the functionally intact subgroup. This variable was called the functional status index.

A morbidity index was generated by ascribing one point to the presence of each of the following chronic illnesses reported by participants at baseline and summing them: depression, diabetes mellitus, stroke, liver disease, Parkinson disease, autoimmune disease, lung disease, heart disease, or cancer. In addition, we controlled for participants' baseline score on the General Health subscale of the SF-36. Marital status was dichotomized into 2 groups: married and living with a spouse and those in any other circumstance, including married and living apart from spouse, widower or widow, divorced, and single/never married.

Descriptive statistics were calculated for baseline variables for the entire cohort. Hierarchical (repeated measures within participants by primary care physician) longitudinal analyses were performed on QWB-SA scores from years 2 through 4 regressed using promising independent variables found in the linear regression models. This analysis 
method (PROC MIXED in SAS software; SAS, Inc., Cary, NC) examines associations between independent variables and the change in the dependent variable over time. Annual changes in confidant status were reflected in the analysis.

To analyze bivariate associations between baseline variables and death, $\chi^{2}$ and $t$ tests were used. Kaplan-Meier analysis was performed to compare unadjusted survival curves; the Wilcoxon statistic was used to assess significance. A Cox proportional hazards model (PROC PHREG in SAS) then was created by including the primary variable of interest (confidant) and by considering covariates such as physical function and comorbid conditions along with promising independent predictors of mortality. Interaction terms of confidant, marital status, and functional status variables were included in the models because of suspected associations. Robust sandwich variance matrix models were used for standard errors and confidence intervals. The variable of age at study entry was found to have time-varying effects on the hazard, violating the assumptions of the proportional hazard model. An interaction term for this variable with survival time was incorporated into the Cox models, as recommended by Allison. ${ }^{12}$ All analyses were performed using SAS 9.2 (version 5.1.2600). Associations with $P<.05$ were considered to be statistically significant.

\section{Results}

Eight hundred fifty-two individuals completed the initial questionnaire. At study entry, 740 $(87 \%)$ said that they had a confidant. Being younger, married, male sex, and having higher income were associated with having a confidant. By year 4 , only $48 \%$ of respondents still reported having a confidant.

Baseline characteristics of the study population are shown in Table 1 . Table 1 also shows associations between confidant status and several baseline measures of functional status, self-reported health, morbidity, and health-related quality of life. Having a confidant was associated with better SF-36 domain scores $(P<.0001)$, self-rated health, QWB-SA scores, and lower morbidity index scores at baseline. We classified 648 participants as functionally intact (total ADL plus IADL scores of $\geq 27$ ), of whom 572 (88\%) had a confidant. Only 204 participants were considered functionally impaired, of whom 168 (82\%) had a confidant.

\section{Associations Between Confidant Status and Quality of Life}

A multivariate, longitudinal growth curve model including all participants was constructed to examine whether having a confidant was associated with changes in quality of well-being (QWB-SA) over the duration of the study (Table 2). Younger age at study entry; better general health; higher functional status; more education; lower morbidity scores; higher SF-36 scores in physical function, energy, and social function; and having a confidant were associated with better quality of life over time. Only the impaired category of the functional status index was associated with poorer longitudinal quality of life.

\section{Associations Between Confidant Status and Length of Life}

As of September 30, 2011, 332 participants (39\%) had died. With respect to mortality, the data set then included up to a maximum of 11.7 calendar years and 7852 person-years of follow-up. Older age, lower body mass index (BMI), lower income, and being unmarried at study entry were associated with greater risk of death. Death also was associated with poorer baseline functional status scores for ADLs and IADLs, general health on the SF-36, self-rated health, and worse QWB-SA scores (Table 1). Having a confidant was not statistically significant $(P=.08)$.

Life-table analysis was performed on the whole cohort to examine the effect of having a confidant on life expectancy. This uncontrolled analysis (Figure 1) also showed no difference in the survival curves for the group with a confidant versus that without a confidant, using the Wilcoxon test for significance $(P=0.16)$. The curves appear to diverge after the tenth year of observation.

Survival time was analyzed for the impact of covariates using Cox proportional hazard models (Table 3). The overall model to assess the contribution of variables to survival time for the cohort showed that female sex, marital status, higher BMI, physical function score, and lower bodily pain score were associated with longer survival. An interaction term of baseline age and survival also was statistically significant, as was the interaction term of confidant and functional status $(P=.024)$. 
Table 1. Characteristics of the Study Population with Respect to Vital Status and Confidant Status

\begin{tabular}{|c|c|c|c|c|c|}
\hline Characteristics & Patients (n) & Alive & $P$ & Confidant & $P$ \\
\hline \multicolumn{6}{|l|}{ Age (years) } \\
\hline $65-74$ & 496 & $361(73 \%)$ & & $430(87 \%)$ & \\
\hline $75-84$ & 302 & $150(50 \%)$ & & $269(89 \%)$ & \\
\hline$\geq 85$ & 53 & $8(15 \%)$ & $<.0001$ & $40(75 \%)$ & .026 \\
\hline \multicolumn{6}{|l|}{ Sex } \\
\hline Men & 365 & $212(58 \%)$ & & $329(90 \%)$ & \\
\hline Women & 484 & $308(63 \%)$ & .13 & $411(84 \%)$ & .014 \\
\hline \multicolumn{6}{|l|}{ Race } \\
\hline White & 723 & $441(61 \%)$ & & $629(87 \%)$ & \\
\hline Nonwhite & 129 & $79(61 \%)$ & .96 & $111(86 \%)$ & .77 \\
\hline \multicolumn{6}{|l|}{ Marital status } \\
\hline Married & 546 & $367(67 \%)$ & & $502(92 \%)$ & \\
\hline Other & 306 & $153(50 \%)$ & $<.0001$ & $238(78 \%)$ & $<.0001$ \\
\hline \multicolumn{6}{|l|}{ Annual income (\$) } \\
\hline$<15,000$ & 162 & $81(50 \%)$ & & $134(83 \%)$ & \\
\hline $15,000-35,000$ & 373 & $220(59 \%)$ & & $320(86 \%)$ & \\
\hline$>35,000$ & 294 & $204(69 \%)$ & .0002 & $267(91 \%)$ & .032 \\
\hline \multicolumn{6}{|l|}{ Education } \\
\hline$<$ High school & 135 & $77(57 \%)$ & & $117(87 \%)$ & \\
\hline High school & 210 & $138(66 \%)$ & & $186(89 \%)$ & \\
\hline$>$ High school & 507 & $202(60 \%)$ & .24 & $437(86 \%)$ & .69 \\
\hline \multicolumn{6}{|l|}{ Confidant } \\
\hline Present & 740 & $460(62 \%)$ & & & \\
\hline Absent & 112 & $60(54 \%)$ & .082 & & \\
\hline \multicolumn{6}{|l|}{ ADL score } \\
\hline $0-13$ & 291 & $144(49 \%)$ & & $246(85 \%)$ & \\
\hline 14 & 561 & $376(67 \%)$ & $<.0001$ & $494(88 \%)$ & .15 \\
\hline \multicolumn{6}{|l|}{ IADL score } \\
\hline $0-13$ & 257 & $108(42 \%)$ & & $215(84 \%)$ & \\
\hline 14 & 595 & $412(69 \%)$ & $<.0001$ & $525(88 \%)$ & .070 \\
\hline \multicolumn{6}{|l|}{ Continuous variables* } \\
\hline Age & 851 & $72.4(4.9)$ & .013 & $74.0(5.8)$ & .19 \\
\hline BMI & 839 & $29.0(5.3)$ & .011 & $28.6(5.5)$ & .91 \\
\hline QWB-SA (0 or 1$)$ & 848 & $0.66(0.12)$ & $<.0001$ & $0.64(0.13)$ & $<.0001$ \\
\hline $\mathrm{ADL}$ & 852 & $13.6(0.94)$ & $<.0001$ & $13.5(1.1)$ & .12 \\
\hline IADL & 852 & $13.6(1.0)$ & $<.0001$ & $13.3(1.5)$ & .19 \\
\hline Self-rated health & 793 & $80.0(14.7)$ & $<.0001$ & $76.9(16.6)$ & .0009 \\
\hline Gait time & 778 & $15.6(4.2)$ & $<.0001$ & $17.0(7.0)$ & .22 \\
\hline Physical function & 846 & $71.8(24.1)$ & $<.0001$ & $65.8(27.4)$ & $<.0001$ \\
\hline Physical limitations & 846 & $62.5(40.1)$ & $<.0001$ & $57.2(42.1)$ & .0003 \\
\hline Emotional limits & 845 & $78.9(34.7)$ & .0029 & $78.2(35.2)$ & $<.0001$ \\
\hline Bodily pain & 846 & $69.4(23.1)$ & .0006 & $68.7(23.8)$ & $<.0001$ \\
\hline Emotional health & 846 & $81.1(15.0)$ & .0023 & $81.2(14.7)$ & $<.0001$ \\
\hline Energy & 846 & $59.6(21.1)$ & $<.0001$ & $57.0(21.6)$ & $<.0001$ \\
\hline Social function & 812 & $88.7(16.9)$ & $<.0001$ & $86.9(18.2)$ & .0009 \\
\hline General health & 848 & $68.6(18.4)$ & $<.0001$ & $65.3(18.7)$ & .0026 \\
\hline Morbidity index & 852 & $1.17(1.04)$ & $<.0001$ & $1.29(1.04)$ & .0068 \\
\hline
\end{tabular}

Bolded values are significant at $P<.05$.

${ }^{*}$ Continuous variable values are presented as mean (SD).

ADL, activity of daily living; IADL, instrumental activity of daily living; BMI, body mass index; QWB-SA, self-administered Quality of Well-Being instrument. 
Table 2. Multivariate, Longitudinal Model for the Self-Administered Quality of Well-Being Instrument

\begin{tabular}{lcc}
\hline & \multicolumn{2}{c}{ Confidant } \\
\cline { 2 - 3 } Independent Variables & Beta (SE) & $P$ \\
\hline Age at study onset & $-0.0024(0.00045)$ & $<.0001$ \\
Sex & $0.0057(0.0056)$ & .30 \\
Body mass index & $0.0018(0.00046)$ & .70 \\
Education category & $0.032(0.0078)$ & $<.0001$ \\
$\quad<12$ grade & $0.012(0.0059)$ & .042 \\
$\quad$ Completed high school & $-0.0082(0.0086)$ & .34 \\
Income category & $-0.0088(0.0056)$ & .12 \\
$\quad<\$ 15,000$ & $-0.040(0.0068)$ & $<.0001$ \\
$\quad \$ 15,000-\$ 35,000$ & $0.00060(0.00018)$ & .0006 \\
Functional status & $-0.0077(0.0025)$ & .0020 \\
General health & $0.016(0.0076)$ & .83 \\
Morbidity index & $0.00098(0.00014)$ & $<.0001$ \\
Race category & $0.00095(0.00016)$ & $<.0001$ \\
Physical function score & $0.00070(0.00017)$ & $<.0001$ \\
Energy & $-0.032(0.0072)$ & $<.0001$ \\
Social function score & & \\
Confidant &
\end{tabular}

\section{Discussion}

Results showed that having a confidant was associated with measures of health-related quality of life. Better longitudinal quality of life was associated with having a confidant, younger age, more education, better health, and having better scores on the SF-36 physical function, bodily pain, and social function domains. These results are consistent with prior studies of the benefits of having a confidant. ${ }^{1,2,6,10,11,13}$

Having a confidant had no independent statistical effect in the survival analyses on life expectancy for the functionally impaired subgroup.
Table 3. Cox Proportional Hazards Model

\begin{tabular}{lcc}
\hline Independent Variables & Hazard Ratio (95\% CI) & $P$ \\
\hline Confidant & NA & .194 \\
Age at study onset & $1.00(0.96-1.04)$ & .91 \\
Sex & $2.35(1.75-3.16)$ & $<.0001$ \\
BMI & $0.95(0.93-0.98)$ & .0001 \\
Income & & \\
$\quad$ Lowest & $1.08(0.73-1.59)$ & .71 \\
$\quad$ Intermediate & $1.27(0.95-1.70)$ & .10 \\
Education & & \\
$\quad$ Lowest & $0.82(0.59-1.15)$ & .25 \\
$\quad$ Intermediate & $0.82(0.63-1.07)$ & .15 \\
Morbidity index & $1.19(1.07-1.31)$ & .0008 \\
Race category & $1.08(0.77-1.52)$ & .69 \\
Married & $\mathrm{NA}$ & .0006 \\
Functional status & $\mathrm{NA}$ & .078 \\
Physical function & $0.98(0.97-0.98)$ & $<.0001$ \\
Bodily pain score & $1.01(1.00-1.01)$ & .0009 \\
Age* survival term & $1.01(1.01-1.02)$ & $<.0001$ \\
Confidant*married & $\mathrm{NA}$ & .89 \\
FuncStatus*married & $\mathrm{NA}$ & .50 \\
FuncStatus*Confidant & $\mathrm{NA}$ & .026 \\
\hline & & \\
& &
\end{tabular}

*Survival is time-linked to age and thus this interaction term has an associated hazard ratio and confidence interval. Hazard ratios and confidence intervals for the confidant, married, and functional status variables are not available because of the use of interaction terms.

$\mathrm{BMI}=$ body mass index.

Longevity was associated with being female, higher BMI, fewer comorbidities, being married, and having better scores on 2 domains of the SF-36 instrument. The interaction term of confidant status and functional status seems to be reasonable because both of these variables are measures of physical and mental capabilities. None of these findings are surprising. Even the

Figure 1. Kaplan-Meier curve survival time by confidant.

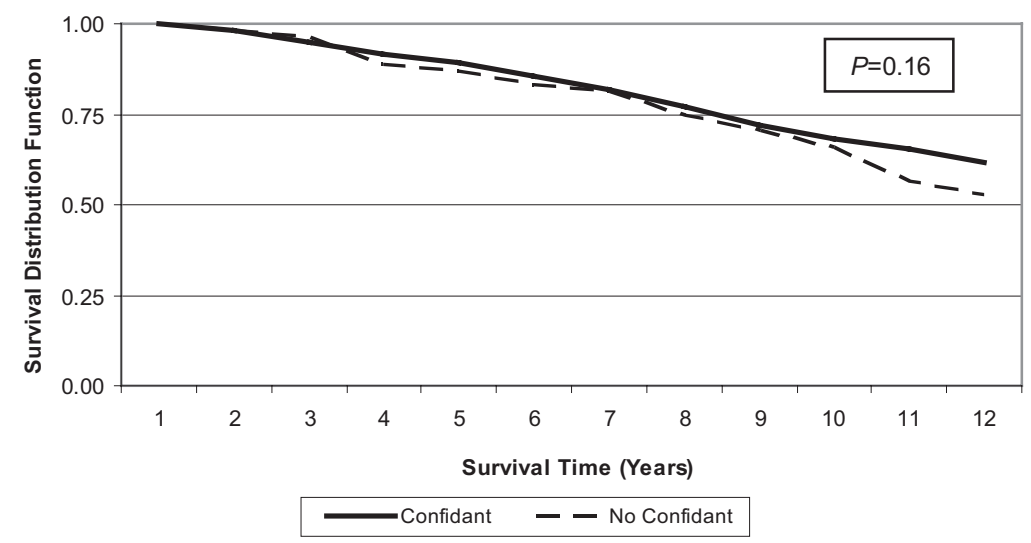


benefit of a higher BMI in the elderly has been reported frequently. ${ }^{14-16}$

Our cohort results differ from the Spanish cohort study ${ }^{6}$ in that confidant status was not significant and that men were more likely to die in our study. The reasons for these differences are not clear. Our results also contrast with those of other authors who reported that loneliness was associated with an increased risk of death. ${ }^{3,5}$ One explanation might be that our cohort may have been younger and that the mortality risks did not emerge until 10 years into the study. Further tracking of our cohort may confirm this hypothesis.

A strength of our study includes its prospective cohort design and the process of controlling for number of illnesses. For the survival analyses, our average period of follow-up was reasonably long and a substantial proportion (39\%) had died. Although the average duration of follow-up for quality of life was relatively short, the participants were all older than age 65, making short-term changes in quality of life more likely. The SSDI has been used in thousands of published studies (eg, Refs. ${ }^{14,16,}$ and ${ }^{17}$ ) and is both less expensive and easier to access and use than the National Death Index. ${ }^{18}$ The SSDI is less complete for younger and foreignborn people, neither of whom was part of our target study population.

This study was, however, limited in several ways. Many of the variables were measured only at the onset of the study. However, 2 important variablesconfidant status and quality of well-being-were measured annually and analyzed in the longitudinal model in that manner. There was a high drop-out rate over the 4 years of the study, resulting in a large number of missing values for health-related quality of life beyond baseline. This reduced our chance to find associations. Nevertheless, the growth curve analysis used all the data that were available. The QWB-SA is fairly sensitive to small changes. We believe that we captured all or nearly all deaths, so those calculations should not have been affected. Severity of illness and its reflection in the patients' need for and experience with certain primary care functions (eg, coordination) are difficult to measure. Even though we used a 2-step process to assess severity of illness, first stratifying and then controlling for number of chronic conditions, similar but not identical to commonly used methods, ${ }^{19,20}$ we are not confident that we removed all the confounding caused by this factor. Charlson and colleagues ${ }^{21}$ have described a more comprehensive measure, but unfortunately we did not have all the data required.

\section{Conclusions}

Having a confidant is significantly associated with measures of health-related quality of life. The relationship between confidant status and death or duration of survival, however, is much more complex. Further measurement within the OKLAHOMA cohort may provide more insight into this relationship.

\section{References}

1. Dickens CM, McGowan L, Percival C, et al. Lack of a close confidant, but not depression, predicts further cardiac events after myocardial infarction. Heart 2004; $90: 518-22$.

2. Mortimer E, Haselow D, Dolan M, et al. Amount of social contact and hip fracture mortality. J Am Geriatr Soc 2008;56:1069-74.

3. Perissinotto CM, Cenzer IS, Covinsky KE. Loneliness in older persons: a predictor of functional decline and death. Arch Int Med 2012;172:1078-83.

4. Bucholz EM, Krumholz HM. Loneliness and living alone. Arch Int Med 2012;172:1084-5.

5. Udell JA, Steg PG, Scirica BA, et al. Living alone and cardiovascular risk in outpatients at risk of or with atherothrombosis. Arch Int Med 2012;172: 1086-95.

6. Rodriguez-Laso A, Zunzunegui MV, Otero A. The effect of social relationships on survival in elderly residents of a Southern European community: a cohort study. BMC Geriatr 2007;7:19.

7. Mold JW, Fryer G, Roberts M. When do older patients change primary care physicians? J Am Board Fam Pract 2004;17:453-60.

8. Mold JW, Vesely SK, Keyl BA, Schenk JB, Roberts $M$. The prevalence, predictors, and consequences of peripheral sensory neuropathy in older patients. J Am Board Fam Pract 2004;17:309-18.

9. Brazier JE, Harper R, Jones NM, et al. Validating the SF-36 health survey questionnaire: new outcome measure for primary care. BMJ 1992;305:160-4.

10. Michael YL, Colditz GA, Coakley E, Kawachi I. Health behaviors, social networks, and healthy aging: cross-sectional evidence from the Nurses' Health Study. Qual Life Res 1999;8:711-22.

11. Newton T, Buckley A, Zurlage M, Mitchell C, Shar A, Woodruff-Borden J. Lack of a close confidant: prevalence and correlates in a medically underserved primary care sample. Psychol Health Med 2008;13: 185-92.

12. Allison PD. Survival Analysis Using SAS: A Practical Guide. 2nd ed. Cary, NC: SAS Institute, Inc.; 2010. 
13. McGhee JL. The influence of qualitative assessments of the social and physical environment on the morale of the rural elderly. Am J Commun Psychol 1984; 12:709-23.

14. Lissin LW, Gauri AJ, Froelicher VF, Ghayoumi A, Myers J, Giacommini J. The prognostic value of body mass index and standard exercise testing in male veterans with congestive heart failure. J Card Fail 2002;8:206-15.

15. Rajpathak SN, Lui Y, Ben-David O, et al. Lifestyle factors of people with exceptional longevity. J Am Geriatr Soc 2011;59:1509-12.

16. Thourani VH, Keeling WB, Kilgo PD, et al. The impact of body mass index on morbidity and short- and long-term mortality in cardiac valvular surgery. J Thorac Cardiovasc Surg 2011;142: $1052-61$.
17. Kelly DL, McMahon RP, Wehring HJ, et al. Cigarette smoking and mortality risk in people with schizophrenia. Schizophr Bull 2011;37:832-8.

18. Hermansen SW, Leitzmann MF, Schatzkin A. The Impact on National Death Index ascertainment of limiting submissions to Social Security Administration Death Master File matches in epidemiologic studies of mortality. Am J Epidemiol 2009;169:901-8.

19. Werner RM, Ash DA. The unintended consequences of publicly reporting quality information. JAMA 2005;293:1239-44.

20. Zhu H, Hill MD. Stroke: the Elixhauser Index for comorbidity adjustment of in-hospital care fatality. Neurology 2008;71:283-7.

21. Charlson ME, Pompei P, Ales KL, MacKenzie CR. A new method of classifying prognostic comorbility inlongitudinal studies: development and validation. J Chronic Dis 1987;40:373-83. 